MATHEMATICS OF COMPUTATION

Volume 74, Number 250, Pages 709-721

S 0025-5718(04)01668-0

Article electronically published on May 11, 2004

\title{
STRICTLY POSITIVE DEFINITE FUNCTIONS ON THE UNIT CIRCLE
}

\author{
XINGPING SUN
}

\begin{abstract}
We study strictly positive definite functions on the unit circle in the Euclidean space of dimension two. We develop several conditions pertaining to the determination of such functions. The major result is obtained by considering the set of real numbers as a vector space over the field of rational numbers and then applying the Kronecker approximation theorem and Weyl's criterion on equidistributions.
\end{abstract}

\section{INTRODUCTION}

Let $S^{m}(m \geq 1)$ denote the unit sphere in the Euclidean space $\mathbb{R}^{m+1}$. Let $x$ and $y$ be two points on $S^{m}$. The usual geodesic distance between the two points is given by

$$
d_{m}(x, y)=\operatorname{Arccos}(x y) .
$$

Here $x y$ denotes the usual inner product of $x$ and $y$. A continuous function $f$ : $[0, \pi] \rightarrow \mathbb{R}$ is said to be positive definite on $S^{m}$ if, for every $N \in \mathbb{N}$ and every set of $N$ points $x_{1}, \ldots, x_{N}$ on $S^{m}$, the $N \times N$ matrix $A$ with $i j$ entry $A_{i j}=\left(f\left(d_{m}\left(x_{i}, x_{j}\right)\right)\right)$ is nonnegative definite, i.e.,

$$
c^{T} A c=\sum_{i=1}^{N} \sum_{j=1}^{N} c_{i} c_{j} f\left(d_{m}\left(x_{i}, x_{j}\right)\right) \geq 0, \quad c=\left(c_{1}, \ldots, c_{N}\right) \in \mathbb{R}^{N} .
$$

If the above inequality becomes strict under the additional hypotheses that the points $x_{1}, \ldots, x_{N}$ are all distinct and that the numbers $c_{1}, \ldots, c_{N}$ are not all zero, we then call the function $f$ strictly positive definite on $S^{m}$. This notion of strict positive-definiteness first appeared in print in a paper by $\mathrm{Xu}$ and Cheney [XC].

Schoenberg [S] characterized all the positive definite functions on $S^{m}$ as those of the form

$$
f(t)=\sum_{k=0}^{\infty} a_{k} P_{k}^{(\lambda)}(\cos t)
$$

in which $\lambda=(m-1) / 2, a_{k} \geq 0$, and $\sum a_{k}<\infty$. Here $P_{k}^{(\lambda)}$ denote the standard Gegenbauer polynomials normalized so that $P_{k}^{(\lambda)}(1)=1$. Gegenbauer polynomials are also called ultraspherical polynomials in the literature; see [Sz, p. 81].

Received by the editor December 9, 2002 and, in revised form, September 29, 2003.

2000 Mathematics Subject Classification. Primary 41A05, 42A15; Secondary 33C45, 33C55.

Key words and phrases. Strict positive-definiteness, the Kronecker approximation, Weyl's criterion, equidistribution. 
An elementary continuity argument shows that the set of all strictly positive definite functions is a proper subset of all positive definite functions. To see whether or not a function $f$ expressed as in equation (1.2) is strictly positive definite, one needs to check that there are "enough" positive coefficients $a_{k}$ in the Gegenbauer polynomial expansion. The key word here is positive, the actual values of the $a_{k}$ 's are immaterial as far as strict positive-definiteness is concerned. For this purpose, we cite the following definition from CMS.

Definition 1.1. Let $\mathbb{Z}_{+}$denote the set of nonnegative integers. A subset $K$ of $\mathbb{Z}_{+}$ is said to induce strict-positive-definiteness (abbreviated as S.P.D.) on $S^{m}$ if the function

$$
t \mapsto \sum_{k \in K} \frac{P_{k}^{(\lambda)}(\cos t)}{2^{k}}
$$

is strictly positive definite on $S^{m}$.

The sequence $2^{-k}$ is chosen here for the sole purpose that the series above converges uniformly. Consequently, any other choice suitable for the purpose will also suffice. Menegatto [M] showed that $K$ must contain infinitely many odd integers as well as infinitely many even ones to induce S.P.D. on $S^{m}$. For $m \geq 2$, this necessary condition has been proved to be also sufficient by Chen, Menegatto, and Sun CMS. Thus, we have the following result: in order for a subset $K \subset \mathbb{Z}_{+}$to induce S.P.D. on $S^{m}(m \geq 2)$, it is necessary and sufficient that $K$ contain infinitely many odd integers as well as infinitely many even ones. This characterization, however, fails to stand for strictly positive definite functions on the unit circle, which corresponds to the case $m=1$. For example, Ron and Sun RS2] showed that the set $\left\{4 k, 4 k+1, k \in \mathbb{Z}_{+}\right\}$does not induce S.P.D. on $S^{1}$.

In this note, we study strictly positive definite functions on $S^{1}$. We first obtain a necessary condition by considering points that are rational multiples of $2 \pi$. The condition asserts that in order that a subset $K \subset \mathbb{Z}_{+}$induce S.P.D. on $S^{1}$, it is necessary that for every natural number $N$ and every $j=0,1, \ldots, N-1$, the intersection $\bar{K} \cap Z_{N}^{j}$ be infinite, where $\bar{K}:=\{ \pm k: k \in K\}$ and $Z_{N}^{j}:=\{k: k \in$ $\mathbb{Z}, k \equiv j(\bmod N)\}$. We then consider the set of real numbers as a vector space over the field of rational numbers, and we use the Kronecker approximation to prove that in order that a subset $K \subset \mathbb{Z}_{+}$induce S.P.D. on $S^{1}$, it is sufficient that for every natural number $N$ and every $j=0,1, \ldots, N-1$, the intersection $K \cap Z_{N}^{j}$ has the Kronecker approximation property; see [A, Theorem 7.10]. The version of the Kronecker approximation theorem that we need in this paper follows from Weyl's criterion on "equidistribution" [W].

There is a gap between the necessary condition and the sufficient one, which we will elaborate upon in the last section of the paper. Giving a full characterization for strictly positive definite functions on the unit circle appears to be a rather challenging task. We have tried very hard but are so far unable to show that the necessary condition that the intersection $\bar{K} \cap Z_{N}^{j}$ be infinite for each natural number $N$ and every $j=0,1, \ldots, N-1$ is also a sufficient condition for the subset $K \subset \mathbb{Z}_{+}$to induce S.P.D. on $S^{1}$. We hope that the challenge will inspire other able mathematicians to devote their energy to this problem. 


\section{EQUivalent CONDITIONS}

When $m=1$, the Gegenbauer polynomials in equation (1.2) are just the Tchebychev polynomials $T_{k}(\cdot)=\cos k\left(\cos ^{-1} \cdot\right)$. Accordingly, the positive definite functions on $S^{1}$ can be expressed in the form

$$
f(t)=\sum_{k=0}^{\infty} a_{k} \cos k t, \quad t \in[0, \pi]
$$

Given two points $x_{1}, x_{2} \in S^{1}$, we identify them with two angles $0<\theta_{1}<\theta_{2} \leq 2 \pi$. Thus

$$
d_{1}\left(x_{1}, x_{2}\right)=\left\{\begin{array}{lr}
\theta_{2}-\theta_{1}, & \text { if } \theta_{2}-\theta_{1} \leq \pi \\
2 \pi-\left(\theta_{2}-\theta_{1}\right), & \text { if } \theta_{2}-\theta_{1}>\pi
\end{array}\right.
$$

Since for each $k=0,1, \ldots$, we have $\cos k\left(2 \pi-\left(\theta_{2}-\theta_{1}\right)\right)=\cos k\left(\theta_{2}-\theta_{1}\right)$, we can work with the angular variable $\theta$ and study positive definite functions on the unit circle in the domain $(0,2 \pi]$. We then use the scaling factor $2 \pi$ and Euler's formula and express the positive definite functions on the unit circle in the form

$$
f(t)=\sum_{k=-\infty}^{\infty} b_{k} \exp (2 \pi i k t), \quad t \in(0,1]
$$

in which $b_{0}=a_{0}$ and $b_{-k}=b_{k}=a_{k} / 2 \geq 0(k>0)$ and $\sum_{k=-\infty}^{\infty} b_{k}<\infty$.

Let $K \subset \mathbb{Z}_{+}$, and let $A$ be a finite subset of $\mathbb{Z}_{+}$. A moment's reflection reveals that $K$ induces S.P.D. on $S^{1}$ if and only if $K \backslash A$ does. In particular, we can interchangeably assume that $K \subset \mathbb{Z}_{+}$or that $K \subset \mathbb{N}$, when deciding whether or not a subset $K \subset \mathbb{Z}_{+}$induces S.P.D. on $S^{1}$.

Theorem 2.1. Let $K \subset \mathbb{N}, \bar{K}:=\{ \pm k: k \in K\}$, and let

$$
g(t):=\sum_{k \in K} 2^{-k} \cos (2 \pi i k t)=\sum_{k \in \bar{K}} 2^{-(|k|+1)} \exp (2 \pi i k t), \quad t \in(0,1] .
$$

Then the following statements are equivalent:

(i) The subset $K$ induces S.P.D. on $S^{1}$.

(ii) For every natural number $N$ and every set of $N$ distinct numbers $\theta_{1}, \ldots, \theta_{N}$ in $(0,1]$, the $N$ functions

$$
\theta \mapsto g\left(\theta-\theta_{j}\right), \quad j=1, \ldots, N
$$

are linearly independent on the set $\left\{\theta_{1}, \ldots, \theta_{N}\right\}$ over the field of real numbers.

(iii) For every natural number $N$ and every set of $N$ distinct numbers $\theta_{1}, \ldots, \theta_{N}$ in $(0,1]$, the $N$ functions

$$
u \mapsto \exp \left(2 \pi i \theta_{j} u\right), \quad j=1, \ldots, N,
$$

are linearly independent on the set $\bar{K}$ over the field of real numbers. 
Proof. To prove the equivalence between parts (i) and (ii), let $\theta_{1}, \ldots, \theta_{N}$ be $N$ $(N \in \mathbb{N})$ distinct numbers in $(0,1]$. By Schoenberg's result [S], we know that the $N \times N$ matrix with $i j$-entry $g\left(\theta_{i}-\theta_{j}\right)$ is nonnegative definite. In order that it be positive definite, it is necessary and sufficient that the matrix be nonsingular, which is equivalent to saying that the $N$ functions

$$
\theta \mapsto g\left(\theta-\theta_{j}\right), \quad j=1, \ldots, N,
$$

are linearly independent on the set $\left\{\theta_{1}, \ldots, \theta_{N}\right\}$ over the field of real numbers.

To prove the equivalence between parts (i) and (iii), we use equation (2.2) and work on the quadratic form

$$
\begin{aligned}
\sum_{i=1}^{N} \sum_{j=1}^{N} c_{i} c_{j} g\left(\theta_{i}-\theta_{j}\right) & =\sum_{k \in \bar{K}} 2^{-(|k|+1)} \sum_{i=1}^{N} \sum_{j=1}^{N} c_{i} c_{j} \exp \left(2 \pi i k\left(\theta_{i}-\theta_{j}\right)\right) \\
& =\sum_{k \in \bar{K}} 2^{-(|k|+1)}\left|\sum_{j=1}^{N} c_{j} \exp \left(2 \pi i k \theta_{j}\right)\right|^{2} .
\end{aligned}
$$

In order that the quadratic form be positive for every nonzero vector $\left(c_{1}, \ldots, c_{N}\right) \in$ $\mathbb{R}^{N}$, it is necessary and sufficient that there be a $k \in \bar{K}$ such that $\sum_{j=1}^{N} c_{j} \exp \left(2 \pi i k \theta_{j}\right)$ $\neq 0$, which is equivalent to saying that the $N$ functions as shown in equation (2.3) are linearly independent on the set $\bar{K}$ over the field of real numbers.

The equivalent condition as stated in part (iii) of Theorem 2.1 is the most useful in this paper. Since for a given $K \subset \mathbb{N}$ and each fixed real number $\theta$, the set of complex numbers $\{\exp (i \theta k), k \in \bar{K}\}$ is closed under the operation of complex number conjugation, we have the following result.

Proposition 2.2. Let $K \subset \mathbb{N}$, and let $\theta_{1}, \ldots, \theta_{N}$ be $N(N \geq 1)$ distinct real numbers. Then the following statements are equivalent:

(i) The $N$ functions as shown in equation (2.3) are linearly independent on the set $\bar{K}:=\{ \pm k: k \in K\}$ over the field of real numbers.

(ii) The $N$ functions as shown in equation (2.3) are linearly independent on the set $K$ over the field of real numbers.

(iii) The $N$ functions as shown in equation (2.3) are linearly independent on the set $\bar{K}$ over the field of complex numbers.

\section{UBIQUitous SETS AND A NECESSARY CONDITION}

Let $K \subset \mathbb{Z}_{+}$. In light of Theorem 2.1, we seek conditions on $K$ so that for every natural number $N$, and every set of $N$ distinct numbers $\theta_{1}, \ldots, \theta_{N}$ in $(0,1]$, the $N$ functions as shown in equation (2.3) are linearly independent on the set $\bar{K}$ over the field of real numbers. In this section, we study the case in which the $N$ distinct numbers $\theta_{1}, \ldots, \theta_{N}$ in $(0,1]$ are all rational.

Definition 3.1. A subset $\Gamma$ of $\mathbb{Z}$ is said to be ubiquitous modulo (or simply ubiquitous), if for every positive integer $N(N \geq 2)$ and every $j(0 \leq j \leq N-1)$, there is a $k \in \Gamma$, such that $k \equiv j(\bmod N)$.

Proposition 3.2. Assume that a subset $\Gamma \subset \mathbb{Z}$ is ubiquitous. Then for every positive integer $N(N \geq 2)$ and every $j(0 \leq j \leq N-1)$, there are infinitely many $k \in \Gamma$, such that $k \equiv j(\bmod N)$. 
Proof. Fix an $N \in \mathbb{N}(N \geq 2)$ and a $j(0 \leq j \leq N-1)$. We show that for every natural number $p$, there is a $k \in \Gamma,|k| \geq p N$, such that $k \equiv j(\bmod N)$. Since $\Gamma$ is ubiquitous, there is a $k \in \Gamma$, such that

$$
k \equiv p N+j \quad(\bmod 3 p N) .
$$

That is, there is a $q \in Z$, such that $k=q \cdot(3 p N)+p N+j=(3 p q+p) N+j$, implying $k \equiv j(\bmod N)$. It is easy to see that $|k| \geq p N$. One then completes the proof by noting that $p$ is an arbitrary natural number.

If a subset $\Gamma$ of $\mathbb{Z}$ contains arbitrarily long strings of consecutive odd integers as well as even ones, then we have the following two results about $\Gamma$ : (i) $\Gamma$ is ubiquitous; (ii) $\Gamma$ induces S.P.D. on $S^{m}$ for all $m=1,2, \ldots$ The first result is obvious, and the second result is proved in [RS1]. The word "ubiquitous" and the result of Proposition 3.2 may have helped in creating an image that the elements of a ubiquitous subset of $\mathbb{Z}_{+}$occur in $\mathbb{Z}_{+}$with a certain "high density". But in fact, one can make a ubiquitous subset of $\mathbb{Z}_{+}$whose elements occur as sparsely in $\mathbb{Z}_{+}$as one wishes. To make this statement more precise, we use the concept of "counting function" as defined in [PS, p. 85]. The counting function $D(r)$ of a sequence $r_{1}, r_{2}, \ldots, r_{n}, \ldots, 0<r_{1} \leq r_{2} \leq \cdots \leq r_{n} \leq \cdots$, is defined as the number of those $r_{n}$ 's that are not greater than $r, r \geq 0$ :

$$
D(r)=\sum_{r_{n} \leq r} 1 .
$$

Generally speaking, the smaller the counting function of a sequence, the faster the sequence approaches infinity.

Proposition 3.3. Let $X$ be any given sequence of positive real numbers that approaches infinity, and let $D_{X}(r)$ be the counting function for $X$. There exists a ubiquitous subset $K$ of $\mathbb{Z}_{+}$with counting function $D_{K}(r)$, such that

$$
D_{K}(r) \leq D_{X}(r), \quad \text { for all } r \geq 0 \text {. }
$$

Proof. For the convenience of writing, we doubly index the elements of $X$ as $x_{n, j}, n=2,3, \ldots, 0 \leq j \leq n-1$, with the order

$$
0 \leq x_{2,0} \leq x_{2,1} \leq x_{3,0} \leq x_{3,1} \leq x_{3,2} \leq \cdots \leq x_{n, 0} \leq x_{n, 1} \leq \cdots \leq x_{n,(n-1)} \leq \cdots .
$$

We use mathematical induction to choose the elements of $K$. Choose $k_{2,0} \in \mathbb{N}$, such that $k_{2,0}>x_{2,0}$ and $k_{2,0} \equiv 0(\bmod 2)$, and choose $k_{2,1} \in \mathbb{N}$, such that $k_{2,1}>\max \left\{x_{2,1}, k_{2,0}\right\}$ and $k_{2,1} \equiv 1(\bmod 2)$. Suppose that for an $n(n \geq 3)$, the elements $k_{(n-1), j}(0 \leq j \leq n-2)$ have been properly chosen. We choose $k_{n, 0} \in \mathbb{N}$ such that $k_{n, 0}>\max \left\{x_{n, 0}, k_{(n-1),(n-2)}\right\}$ and $k_{n, 0} \equiv 0(\bmod n)$. Then we successively choose $k_{n, j} \in \mathbb{N}, 1 \leq j \leq n-1$, so that $k_{n, j}>\max \left\{x_{n, j}, k_{n,(j-1)}\right\}$ and $k_{n, j} \equiv j(\bmod n)$. We therefore obtain the sequence

$$
0<k_{2,0}<k_{2,1}<k_{3,0}<k_{3,1}<k_{3,2}<\cdots<k_{n, 0}<k_{n, 1}<\cdots<k_{n,(n-1)}<\cdots .
$$

Denote this sequence by $K$. It then follows that the set $K$ is ubiquitous and that equation (3.1) holds true.

Lemma 3.4. Let $N$ be a natural number, and let $\theta_{j}:=p_{j} / q_{j}(1 \leq j \leq N)$ be $N$ distinctive rational numbers in $(0,1]$. Let $Q(\geq N)$ be a common multiple of $q_{j}(1 \leq j \leq N)$. Let $\Gamma \subset \mathbb{Z}$. Assume that for each $l=0,1, \ldots, N-1$, there is a $k \in \Gamma$, such that $k \equiv l(\bmod Q)$. Then the $N$ functions as shown in equation (2.3) are linearly independent on $\Gamma$ over the field of complex numbers. 
Proof. Suppose that $c_{1}, \ldots, c_{N}$ are $N$ complex numbers such that

$$
\sum_{j=1}^{N} c_{j} \exp \left(2 \pi i k \theta_{j}\right)=0, \quad \text { for all } k \in \Gamma .
$$

We show that all the $c_{j}$ 's are zero. For each $l=0,1, \ldots, N-1$, select a $k \in \Gamma$, such that $k \equiv l(\bmod Q)$. That is, $k=s Q+l$, where $s$ is an integer. We then have, for each $l=0,1, \ldots, N-1$,

$$
\sum_{j=1}^{N} c_{j} \exp \left(2 \pi i k \theta_{j}\right)=\sum_{j=1}^{N} c_{j} \exp \left(2 \pi i(s Q+l) \cdot \frac{p_{j}}{q_{j}}\right)=\sum_{j=1}^{N} c_{j} \exp \left(2 \pi i l \cdot \frac{p_{j}}{q_{j}}\right)=0 .
$$

The $N \times N$ Vandermonde matrix with $j l$-entries $\exp \left(2 \pi i l \cdot \frac{p_{j}}{q_{j}}\right)(1 \leq j \leq N, 0 \leq l \leq$ $N-1)$ and $N$ distinct complex numbers $\exp \left(2 \pi i \cdot \frac{p_{j}}{q_{j}}\right)(1 \leq j \leq N)$ in the second row has nonzero determinant. Therefore all the $c_{j}$ 's are zero.

Theorem 3.5. Let $\Gamma \subset \mathbb{Z}$. The following two statements are equivalent:

(i) For every natural number $N$ and every set of $N$ distinct rational numbers $\theta_{j}(j=1, \ldots, N)$ in $(0,1]$, the $N$ functions as shown in equation (2.3) are linearly independent on $\Gamma$ over the field of complex numbers.

(ii) The subset $\Gamma$ of $\mathbb{Z}$ is ubiquitous.

Proof. It is a direct consequence of Lemma 3.4 that part (ii) implies part (i). To show that part (i) implies part (ii), assume that $\Gamma$ is not ubiquitous. Then there is a positive integer $N>1$ and a nonnegative integer $l(0 \leq l \leq N-1)$ for which there is no $k \in \Gamma$ such that $k \equiv l(\bmod N)$. For $j=1, \ldots, N$, let $c_{j}:=\exp \left(-2 \pi i \frac{l j}{N}\right)$ and $\theta_{j}:=\frac{j}{N}$. Write each $k \in K$ in the form $k=\mu N+\nu$, where $\nu \neq l$. We then have

$$
\begin{aligned}
\sum_{j=1}^{N} c_{j} \exp \left(2 \pi i k \theta_{j}\right) & \\
= & \sum_{j=1}^{N} \exp \left(-2 \pi i \frac{l j}{N}\right) \cdot \exp \left(2 \pi i(\mu N+\nu) \frac{j}{N}\right) \\
& =\sum_{j=1}^{N} \exp \left(2 \pi i\left(\frac{\nu}{N}-\frac{l}{N}\right) j\right) \\
& =\exp \left(2 \pi i\left(\frac{\nu-l}{N}\right)\right) \cdot \frac{1-\exp \left(2 \pi i\left(\frac{\nu-l}{N}\right) N\right)}{1-\exp \left(2 \pi i\left(\frac{\nu-l}{N}\right)\right)}=0 .
\end{aligned}
$$

Corollary 3.6. Let $K \subset \mathbb{Z}_{+}$. In order that $K$ induce S.P.D. on $S^{1}$, it is necessary that $\bar{K}$ be ubiquitous.

Proof. Suppose that $\bar{K}$ is not ubiquitous. Then by Theorem 3.5, there exist $N$ ( $N \geq$ 2 ) distinct rational numbers $\theta_{j}$ in $(0,1]$ such that the $N$ functions as shown in equation (2.3) are linearly dependent on $\bar{K}$ over the field of complex numbers. By Proposition 2.2 and Theorem 2.1, the subset $K \subset \mathbb{Z}_{+}$does not induce S.P.D. on $S^{1}$. 


\section{KRONECKER APPROXIMATION AND EQUIDISTRIBUTION}

We consider the set of all real numbers as a vector space over the field of rational numbers. Let $\tau_{1}, \ldots, \tau_{M}$ be $M$ arbitrary real numbers. If there exist $M$ rational numbers $r_{1}, \ldots, r_{M}$, not all zero, such that $r_{1} \tau_{1}+\cdots+r_{M} \tau_{M}=0$, then we say that $\tau_{1}, \ldots, \tau_{M}$ are linearly dependent over the field of rational numbers. Otherwise, we say that $\tau_{1}, \ldots, \tau_{M}$ are linearly independent over the field of rational numbers.

Definition 4.1. Let $\Gamma$ be a subset of $\mathbb{Z}$. If $\tau_{1}, \ldots, \tau_{M}$ are arbitrary real numbers, if $\theta_{1}, \ldots, \theta_{M}$ are irrational numbers such that the $(M+1)$ numbers $1, \theta_{1}, \ldots, \theta_{M}$ are linearly independent over the field of rational numbers, and if for every given $\epsilon>0$ there are an integer $k \in \Gamma$ and integers $m_{1}, \ldots, m_{M}$ such that

$$
\left|k \theta_{j}-m_{j}-\tau_{j}\right|<\epsilon \text { for } j=1, \ldots, M,
$$

then we say that $\Gamma$ has the Kronecker approximation property.

The well-known Kronecker approximation theorem (e.g., [A p. 154]) asserts that the set $\mathbb{Z}_{+}$has the Kronecker approximation property. Exercise 7 in Chapter 7 in [A, p. 160] asserts that for an arbitrary large (fixed) natural number $N$, the set $\{N, N+1, \ldots\}$ has the Kronecker approximation property. In the theory of random variables, the stronger notion "equidistribution" (or uniform distribution) prevails. A sequence in $M$ dimensions $\tau^{(n)}=\left(\tau_{1}^{(n)}, \ldots, \tau_{M}^{(n)}\right)$ is equidistributed in the unit cube

$$
C_{M}: 0 \leq \tau_{1} \leq 1, \ldots, 0 \leq \tau_{M} \leq 1
$$

if for $0 \leq a_{j}<b_{j} \leq 1(j=1, \ldots, M)$,

$$
\lim _{N \rightarrow \infty} \frac{1}{N} \sum_{\substack{a_{j} \leq \tau_{j}^{(n)} \leq b_{j}(j=1, \ldots, M) \\ 1 \leq n \leq N}} 1=\prod_{j=1}^{M}\left(b_{j}-a_{j}\right) .
$$

Let $\operatorname{frac}\{x\}:=x-\lfloor x\rfloor$ denote the fractional part of $x$. Let $\Gamma \subset \mathbb{Z}$. If the $M$-dimensional sequence $\left(\left\{k \theta_{1}\right\}, \ldots,\left\{k \theta_{M}\right\}\right)(k \in \Gamma)$ is equidistributed in $C_{M}$, then the sequence is automatically dense in $C_{M}$, and therefore $\Gamma$ has the Kronecker approximation property. It is reasonable to think that there is a $\Gamma \subset \mathbb{Z}$ that has the Kronecker approximation property, but the $M$-dimensional sequence (frac $\left.\left\{k \theta_{1}\right\}, \ldots, \operatorname{frac}\left\{k \theta_{M}\right\}\right)(k \in \Gamma)$ is not equidistributed in $C_{M}$. Nevertheless, we are unable to find such an example in the literature.

In this paper, we only need the Kronecker approximation property. However, it appears that equidistribution is more extensively studied in the literature. The celebrated Weyl's criterion [W] asserts that the $M$-dimensional sequence $\tau^{(n)}=$ $\left(\tau_{1}^{(n)}, \ldots, \tau_{M}^{(n)}\right)$ is equidistributed in $C_{M}$ if and only if

$$
\lim _{N \rightarrow \infty} \frac{1}{N} \sum_{n=1}^{N} \exp \left(2 \pi i\left(k_{1} \tau_{1}^{(n)}+\cdots+k_{M} \tau_{M}^{(n)}\right)\right)=0
$$

for every set of integers $k_{1}, \ldots, k_{M}$ not all zero. Weyl [W] also proved that "polynomial" sequences of the form

$$
x_{n}:=\operatorname{frac}\left\{\alpha n^{q}+a_{1} n^{q-1}+\cdots+a_{q}\right\},
$$

$\alpha$ is an irrational number, and $a_{1}, \ldots, a_{q}$ are real numbers,

are equidistributed in $C_{1}:=[0,1]$. As an easy consequence of Weyl's results, we have the following: 
Theorem 4.2. Suppose that $\theta_{1}, \ldots, \theta_{M}$ are $M$ real numbers so that the $(M+1)$ numbers $1, \theta_{1}, \ldots, \theta_{M}$ are linearly independent over the field of rational numbers. Let $p(n):=a_{0} n^{q}+a_{1} n^{q-1}+\cdots+a_{q}$ be a polynomial of degree $q(q \geq 1)$ with integer coefficients. Then the $M$-dimensional sequence

$$
\left(\operatorname{frac}\left\{p(n) \theta_{1}\right\}, \ldots, \operatorname{frac}\left\{p(n) \theta_{M}\right\}\right)
$$

is equidistributed in $C_{M}$.

Proof. By the sufficiency part of Weyl's criterion, it suffices to show that, for every set of integers $k_{1}, \ldots, k_{M}$, not all zero,

$$
\lim _{N \rightarrow \infty} \frac{1}{N} \sum_{n=1}^{N} \exp \left(2 \pi i\left(k_{1} p(n) \theta_{1}+\cdots+k_{M} p(n) \theta_{M}\right)\right)=0 .
$$

Since the $(M+1)$ real numbers $1, \theta_{1}, \ldots, \theta_{M}$ are linearly independent over the field of rational numbers, the number $k_{1} \theta_{1}+\cdots+k_{M} \theta_{M}$ is irrational; therefore the polynomial sequence

$$
y_{n}:=\operatorname{frac}\left\{k_{1} p(n) \theta_{1}+\cdots+k_{M} p(n) \theta_{M}\right\}
$$

is equidistributed in $C_{1}$. Using the necessity part of the one-dimensional version of Weyl's criterion, we see that equation (4.1) is true.

\section{A SUFFICIENT CONDITION}

Theorem 5.1. Let $K$ be a subset of $\mathbb{Z}_{+}$. In order that $K$ induce S.P.D. on $S^{1}$, it is sufficient that for every $N \geq 2$ and every $j=0,1, \ldots, N-1$ the set $\bar{K} \cap Z_{N}^{j}$ have the Kronecker approximation property.

Proof. Let $(N+1)$ distinct real numbers $\theta_{0}, \theta_{1}, \ldots, \theta_{N}$ in $(0,1]$ be given. We will show that the $(N+1)$ functions

$$
u \mapsto \exp \left(2 \pi i \theta_{j} u\right), \quad j=0,1, \ldots, N,
$$

are linearly independent on the set $\bar{K}$ over the field of real numbers. Equivalently, assume that there are $(N+1)$ real numbers $c_{j}(j=0,1, \ldots, N)$ such that

$$
\Phi(k):=\sum_{j=0}^{N} c_{j} \exp \left(2 \pi i k \theta_{j}\right)=0, \quad k \in \bar{K} .
$$

We show that $c_{0}=c_{1}=\cdots=c_{N}=0$. Rotating the unit circle if necessary, we can assume $\theta_{0}=1$. Recall that the set of real numbers is a vector space over the field of rational numbers. Consider the $(N+1)$ real numbers $\theta_{0}, \theta_{1}, \ldots, \theta_{N}$ as vectors in this space, and assume that they span a subspace $\Theta$ of dimension $(M+1)(0 \leq M \leq N)$. We need to discuss three cases for $M$ : (i) $M=0$; (ii) $M=N$; (iii) $0<M<N$. 
Case (i). In this case, all the numbers $\theta_{0}, \theta_{1}, \ldots, \theta_{N}$ are rational, which is a case covered by Theorem 3.5. Note that the Kronecker approximation property is not needed there.

Case (ii). When $M=N$, the numbers $1, \theta_{1}, \ldots, \theta_{N}$ are linearly independent over the field of rational numbers. The set $\bar{K}$ has the Kronecker approximation property. Therefore for each $n$-tuple $\left(\tau_{1}, \ldots, \tau_{N}\right) \in C_{N}$, there is a sequence $\left\{k_{\gamma}\right\} \subset$ $\bar{K}$, such that $\lim _{\gamma \rightarrow \infty} \operatorname{frac}\left\{k_{\gamma} \theta_{j}\right\}=\tau_{j}(1 \leq j \leq N)$. This implies that

$$
\begin{aligned}
c_{0}+\sum_{j=1}^{N} c_{j} \exp \left(2 \pi i \tau_{j}\right) & =\lim _{\gamma \rightarrow \infty}\left(c_{0}+\sum_{j=1}^{N} c_{j} \exp \left(2 \pi i \cdot \operatorname{frac}\left\{k_{\gamma} \theta_{j}\right\}\right)\right) \\
& =\lim _{\gamma \rightarrow \infty}\left(c_{0}+\sum_{j=1}^{N} c_{j} \exp \left(2 \pi i k_{\gamma} \theta_{j}\right)\right)=0 .
\end{aligned}
$$

Since the $(N+1)$ functions defined on $C_{N}$,

$$
\left(\tau_{1}, \ldots, \tau_{N}\right) \mapsto 1, \quad\left(\tau_{1}, \ldots, \tau_{N}\right) \mapsto \exp \left(2 \pi i \tau_{j}\right), \quad j=1, \ldots, N
$$

are linearly independent on $C_{M}$ over the field of real numbers, we conclude that all the $c_{j}$ 's are zero.

Case (iii). By reordering the set $\left\{1, \theta_{1}, \ldots, \theta_{N}\right\}$, we may assume that $1, \theta_{1}, \ldots$, $\theta_{M}$ form a basis for the subspace $\Theta$, and we express $\theta_{M+1}, \ldots, \theta_{N}$ in linear combinations of $1, \theta_{1}, \ldots, \theta_{M}$ with rational coefficients. We also adapt the equivalent relation " $\equiv "$ on the set $\left\{\theta_{j}, j=0,1, \ldots, N\right\}: \theta_{i} \equiv \theta_{j}$ if and only if $\theta_{i}-\theta_{j}$ is rational. Since $1, \theta_{1}, \ldots, \theta_{M}$ are linearly independent over the field of rational numbers, each of them represents a distinctive equivalence class. Let us assume that there is a total of $L(L \geq M+1)$ equivalence classes. Each element of the set $\left\{\theta_{M+1}, \ldots, \theta_{N}\right\}$ is in exactly one of the $L$ equivalence classes. Reordering the elements of the set $\left\{\theta_{M+1}, \ldots, \theta_{N}\right\}$ while necessary, we can designate $L+1$ natural numbers $E_{0}, E_{1}, \ldots, E_{L}, M+1=E_{0} \leq E_{1} \leq \cdots \leq E_{L}=N+1$ so that for each $n(0 \leq n \leq L-1)$, all the numbers in the set $\left\{\theta_{j}: E_{n} \leq j<E_{n+1}\right\}$ belong to the same equivalence class. If $E_{n}=E_{n+1}$ for some $n(0 \leq n \leq L-1)$, then we consider the set $\left\{\theta_{j}: E_{n} \leq j<E_{n+1}\right\}$ empty. Therefore we may write the numbers of the set $\left\{\theta_{M+1}, \ldots, \theta_{N}\right\}$ in the following forms:

$$
\begin{aligned}
& \theta_{j}=p_{j} / q_{j}+\theta_{n}, \quad \text { if } \quad E_{n} \leq j<E_{n+1}(0 \leq n \leq M), \\
& \theta_{j}=p_{j} / q_{j}+\sum_{s=1}^{M} r_{s n} \theta_{s}, \quad \text { if } \quad E_{n} \leq j<E_{n+1}(M+1 \leq n \leq L-1) .
\end{aligned}
$$

Here $p_{j}, q_{j}\left(q_{j} \neq 0\right)(M+1 \leq j \leq N)$ are integers, and $r_{s n}(1 \leq s \leq M, M+1 \leq$ $n \leq L-1$ ) are rational numbers. Furthermore, as $M$-dimensional vectors in $\mathbb{R}^{M}$, the following are all distinct:

$$
(0, \ldots, 0),(1,0, \ldots, 0), \ldots,(0,0, \ldots, 1),\left(r_{1 n}, \ldots, r_{M n}\right)(M+1 \leq n \leq L-1) .
$$


Using equation (5.1), we write

$$
\begin{aligned}
\Phi(k)=c_{0} & +\sum_{j=1}^{M} c_{j} \exp \left(2 \pi i k \theta_{j}\right) \\
& +\sum_{n=0}^{L-1} \sum_{j=E_{n}}^{E_{n+1}-1} c_{j} \exp \left(2 \pi i k \theta_{j}\right) \\
=c_{0} & +\sum_{j=1}^{M} c_{j} \exp \left(2 \pi i k \theta_{j}\right) \\
& +\sum_{n=0}^{M} \sum_{j=E_{n}}^{E_{n+1}-1} c_{j} \exp \left(2 \pi i k\left(\theta_{n}+\frac{p_{j}}{q_{j}}\right)\right) \\
& +\sum_{n=M+1}^{L-1} \sum_{j=E_{n}}^{E_{n+1}-1} c_{j} \exp \left(2 \pi i k\left(\sum_{s=1}^{M} r_{s n} \theta_{s}+\frac{p_{j}}{q_{j}}\right)\right) \\
=c_{0} & +\sum_{j=1}^{M} c_{j} \exp \left(2 \pi i k \theta_{j}\right) \\
& +\sum_{n=0}^{M}\left[\sum_{j=E_{n}}^{E_{n+1}-1} c_{j} \exp \left(2 \pi i k \frac{p_{j}}{q_{j}}\right)\right] \cdot \exp \left(2 \pi i k \theta_{n}\right) \\
& +\sum_{n=M+1}^{L-1}\left[\sum_{j=E_{n}}^{E_{n+1}-1} c_{j} \exp \left(2 \pi i k \frac{p_{j}}{q_{j}}\right)\right] \cdot \exp \left(2 \pi i k \sum_{s=1}^{M} r_{s n} \theta_{s}\right) \\
= & \sum_{n=0}^{M}\left[c_{n}+\sum_{j=E_{n}}^{E_{n+1}-1} c_{j} \exp \left(2 \pi i k \frac{p_{j}}{q_{j}}\right)\right] \cdot \exp \left(2 \pi i k \theta_{n}\right) \\
& \sum_{j=1}^{L-1}\left[\sum_{j=E_{n}}^{E_{n+1}-1} c_{j} \exp \left(2 \pi i k \frac{p_{j}}{q_{j}}\right)\right] \cdot \exp \left(2 \pi i k \sum_{s=1}^{M} r_{s n} \theta_{s}\right) .
\end{aligned}
$$

Let $Q:=\operatorname{lcm}\left\{q_{M+1}, \ldots, q_{N}\right\}$. By the hypothesis, for each $l(0 \leq l \leq Q-1)$, the set $\bar{K} \cap Z_{Q}^{l}$ has the Kronecker approximation property. Since the $(M+1)$ numbers $1, \theta_{1}, \ldots, \theta_{M}$ are linearly independent over the field of rational numbers, for each $\left(\tau_{1}, \ldots, \tau_{M}\right) \in C_{M}$, there is a sequence

$$
\left\{k_{\gamma}\right\}_{\gamma=1}^{\infty} \subset \bar{K} \cap Z_{Q}^{l}
$$

such that

$$
\lim _{\gamma \rightarrow \infty} \operatorname{frac}\left\{k_{\gamma} \theta_{j}\right\}=\tau_{j}, \quad j=1,2, \ldots, M .
$$

Our intention is to take the limit in equation (5.2). However, the existence of $\lim _{\gamma \rightarrow \infty} \Phi\left(k_{\gamma}\right)$ as in the present form is uncertain. On one hand, we have for each fixed $j(M+1 \leq j \leq N)$ and all $k_{\gamma}$,

$$
\exp \left(2 \pi i k_{\gamma} \cdot\left(p_{j} / q_{j}\right)\right)=\exp \left(2 \pi i l \cdot\left(p_{j} / q_{j}\right)\right) .
$$


On the other hand, the sequence $\exp \left(2 \pi i k \sum_{s=1}^{M} r_{s n} \theta_{s}\right)$ may not have a limit as $\gamma \rightarrow \infty$. To circumvent the difficulty, we write

$$
\begin{aligned}
& \exp \left(2 \pi i k_{\gamma} \sum_{s=1}^{M} r_{s n} \theta_{s}\right) \\
& \quad=\exp \left(2 \pi i \sum_{s=1}^{M} r_{s n}\left(\left\lfloor k_{\gamma} \theta_{s}\right\rfloor+\operatorname{frac}\left\{k_{\gamma} \theta_{s}\right\}\right)\right) \\
& \quad=\exp \left(2 \pi i \sum_{s=1}^{M} r_{s n}\left\lfloor k_{\gamma} \theta_{s}\right\rfloor\right) \cdot \exp \left(2 \pi i \sum_{s=1}^{M} r_{s n} \operatorname{frac}\left\{k_{\gamma} \theta_{s}\right\}\right) .
\end{aligned}
$$

We see that for each $n(M+1 \leq n \leq N)$, the set consisting of the numbers

$$
\exp \left(2 \pi i \sum_{s=1}^{M} r_{s n}\left\lfloor k_{\gamma} \theta_{s}\right\rfloor\right), \quad \gamma=1, \ldots, \infty,
$$

is finite. Therefore we can repeatedly apply the Pigeon Hole Principle to pick infinitely many $k_{\gamma_{\mu}} \in\left\{k_{\gamma}\right\}_{\gamma=1}^{\infty}$ so that for each $n(M+1 \leq n \leq N)$, there is a fixed rational number $R_{n}$, such that

$$
\exp \left(2 \pi i \sum_{s=1}^{M} r_{s n}\left\lfloor k_{\gamma_{\mu}} \theta_{s}\right\rfloor\right)=\exp \left(2 \pi i R_{n}\right)
$$

for all $k_{\gamma_{\mu}}$. Hence, setting $k=k_{\gamma_{\mu}}$ in equation (5.2) and letting $\mu \rightarrow \infty$, we have

$$
\begin{aligned}
& \lim _{\mu \rightarrow \infty} \Phi\left(k_{\gamma_{\mu}}\right) \\
&= 0 \\
&= {\left[c_{0}+\sum_{j=E_{0}}^{E_{1}-1} c_{j} \exp \left(2 \pi i l \frac{p_{j}}{q_{j}}\right)\right] } \\
&+\sum_{n=1}^{M}\left[c_{n}+\sum_{j=E_{n}}^{E_{n+1}-1} c_{j} \exp \left(2 \pi i l \frac{p_{j}}{q_{j}}\right)\right] \cdot \exp \left(2 \pi i \tau_{n}\right) \\
&+\sum_{n=M+1}^{L-1}\left[\sum_{j=E_{n}}^{E_{n+1}-1} c_{j} \exp \left(2 \pi i l \frac{p_{j}}{q_{j}}\right)\right] \cdot \exp \left(2 \pi i R_{n}\right) \cdot \exp \left(2 \pi i \sum_{s=1}^{M} r_{s n} \tau_{s}\right) .
\end{aligned}
$$

Consider the $L$ functions $g_{0}, \ldots, g_{L-1}$ defined on $C_{M}$,

$$
g_{n}: \tau:=\left(\tau_{1}, \ldots, \tau_{M}\right) \mapsto \exp \left(2 \pi i \tau \omega_{n}\right),
$$

where $\omega_{0}=(0, \ldots, 0), \omega_{1}=(1,0, \ldots, 0), \ldots, \omega_{M}=(0,0, \ldots, 1)$, and $\omega_{n}=$ $\left(r_{1 n} \ldots, r_{M n}\right)$ for $n$ with $M+1 \leq n \leq L-1$. Since the $L$ functions as shown in equation (5.3) are linearly independent on $C_{M}$ over the field of complex numbers, we have, for each $n=0,1, \ldots, M$,

$$
c_{n}+\sum_{j=E_{n}}^{E_{n+1}-1} c_{j} \exp \left(2 \pi i l \frac{p_{j}}{q_{j}}\right)=0
$$


and for each $n=M+1, \ldots, L-1$,

$$
\sum_{j=E_{n}}^{E_{n+1}-1} c_{j} \exp \left(2 \pi i l \frac{p_{j}}{q_{j}}\right)=0 .
$$

Note that for each $n=0,1, \ldots, M$, the rational numbers $1, p_{j} / q_{j}\left(E_{n} \leq j<\right.$ $\left.E_{n+1}-1\right)$ are all distinct, and for each $n=M+1, \ldots, L-1$, the rational numbers $p_{j} / q_{j}\left(E_{n} \leq j<E_{n+1}-1\right)$ are also distinct. Also note that equations (5.4a) and (5.4b) hold true for all $l=0,1, \ldots, Q-1$. Therefore we can use Lemma 3.4 to conclude that all the $c_{j}$ 's are zero.

Corollary 5.2. For each fixed $M \in \mathbb{N}, M \geq 2$, let

$$
K:=\left\{k M+j: 0 \leq j \leq\left\lfloor\frac{M}{2}\right\rfloor, k \in \mathbb{N}\right\} .
$$

Then $K$ induces S.P.D. on $S^{1}$.

Proof. By Theorem 5.1, it suffices to prove that for each fixed $N \in \mathbb{N}(N \geq 2)$ and each fixed $j(0 \leq j \leq N-1)$, the set $\bar{K} \cap Z_{N}^{j}$ has the Kronecker approximation property. In fact, if $j \equiv i(\bmod M)$ for $0 \leq i \leq\left\lfloor\frac{M}{2}\right\rfloor$, i.e., there is an $s \in \mathbb{Z}$ such that $j=s M+i$, then for each $k \in \mathbb{N}$, the natural number $k M N+j \in Z_{N}^{j}$. Writing $k M N+j=k M N+s M+i=(k N+s) M+i$, we see that $k M N+j \in K$. Therefore $k M N+j \in \bar{K} \cap Z_{N}^{j}$. For the fixed $M, N$ and $j$, the set $\{k M N+j: k \in \mathbb{N}\}$ has the Kronecker approximation property by Theorem 4.2. If $j \equiv i(\bmod M)$ for $\left\lfloor\frac{M}{2}\right\rfloor<i \leq N-1$, then for each $k \in K,-k M N+j \in Z_{N}^{j}$. Writing $-k M N+j=$ $-k M N+s M+i=-(k N-s-1) M-(M-i)$, we see that $-k M N+j \in \bar{K}$. Therefore $-k M N+j \in \bar{K} \cap Z_{N}^{j}$. Again, for the fixed $M, N$ and $j$, the set $\{-k M N+j: k \in \mathbb{N}\}$ has the Kronecker approximation property.

Some special cases of Corollary 5.2 have been proved by Ron and Sun RS2. For instance, they show that the set $\{3 k, 3 k+1: k \in \mathbb{N}\}$ induces S.P.D. on $S^{1}$. Using Theorem 4.2 and Theorem 5.1, we can construct subsets of $\mathbb{Z}_{+}$of "polynomial growth" that induce S.P.D. on $S^{1}$. For instance, one can start with a polynomial $P$ of degree no less than one with integer coefficients and make a subset $\Gamma$ of $\mathbb{Z}$ in the form $\Gamma:=\left\{P^{k}(n)+k: n \in \mathbb{N} ; 0 \leq k \leq n-1\right\}$. One shows that for every positive integer $N \geq 2$ and every $j=0,1, \ldots, N-1$, the set $\Gamma \cap Z_{N}^{j}$ has the Kronecker approximation property, and therefore, the set $\Gamma \cap \mathbb{Z}_{+}$induces S.P.D. on $S^{1}$.

There is a gap between our sufficient condition (Theorem 5.1) and the necessary condition (Corollary 3.6). The necessary condition requires that the set $\bar{K}$ be ubiquitous, which, by Proposition 3.2, means that for every positive integer $N \geq 2$ and every $j=0,1, \ldots, N-1$, the set $\bar{K} \cap Z_{N}^{j}$ is infinite. The sufficient condition, however, needs each set $\bar{K} \cap Z_{N}^{j}$ to have the Kronecker approximation property. There are abundant examples in the literature that show that infinite subsets of $\mathbb{Z}$ do not have the Kronecker approximation property. An interesting example was given by Polya and Szego [PS, Part 2, Problem 171]: the sequence $n ! e-\lfloor n ! e\rfloor$ has only one accumulation point: zero. From another perspective, we do not see that Theorem 5.1 implies the known result that every subset of $\mathbb{Z}_{+}$that contains arbitrarily long strings of consecutive odd integers as well as consecutive even ones induce S.P.D. on $S^{1}$; see [RS1. This may suggest that some algebraic methods are needed in order to fully characterize strictly positive definite functions on $S^{1}$. 
Despite repeated failures, the author is still motivated to show that $\bar{K}$ ubiquitous is sufficient for $K$ to induce S.P.D. on $S^{1}$.

\section{ACKNOWLEDGMENTS}

The authors has benefited from several fruitful discussions with Professors Debao Chen, Ward Cheney, Les Reid, and Amos Ron on various occasions during the process of writing the paper. In particular, Professors Chen, Cheney, and Ron allowed the author, in a gracious manner, to use some of their unpublished notes stemming from previous collaborations. Professor Reid suggested the word "ubiquitous" in Section 3, and more importantly, he constructed several ingenious "counterexamples" that prevented the author from pursuing a couple of previous misguided approaches.

\section{REFERENCES}

[A] T. M. Apostol, Modular functions and Dirichlet series in number theory, second edition, Springer-Verlag, New York, 1989. MR 90j:11001

[CMS] D. Chen, V. A. Menegatto, and X. Sun, A necessary and sufficient condition for strictly positive definite functions on spheres, Proc. Amer. Math. Soc. 131 (2003), 2733-2740.

$[\mathrm{M}] \quad$ V. A. Menegatto, Strictly positive definite functions on spheres, Ph. D. Dissertation, University of Texas-Austin, 1992.

[PS] G. Polya, and G. Szego, Problems and Theorems in Analysis, Vol. 1, Springer-Verlag, New York, Heidelberg, Berlin, 1972. MR 49:8782

[RS1] A. Ron and X. Sun, Strictly positive definite functions on spheres in Euclidean spaces, Math. Comp. 65 (1996), 1513-1530. MR 97a:41032

[RS2] A. Ron and X. Sun, Strictly positive definite functions on spheres, CMS TR 94-6, University of Wisconsin-Madison, February 1994.

[S] I. J. Schoenberg, Positive definite functions on spheres, Duke Math. J. 9 (1942), 96-108. MR 3:232c

[Sz] Gabor Szegö, Orthogonal Polynomials, Amer. Math. Colloq. Publ., vol. 23, Amer. Math. Soc., Providence, RI, 1959. MR 1:14b

[W] H. Weyl, Über die Gleichverteilung von Zahlen modulo Eins, Math. Ann. 77 (1916), 313352.

[XC] Yuan Xu and E. W. Cheney, Strictly positive definite functions on spheres, Proc. Amer. Math. Soc. 116 (1992), 977-981. MR 93b:43005

Department of Mathematics, Southwest Missouri State University, Springfield, MisSOURI 65804

E-mail address: xis280f@smsu.edu 